\title{
Gobernanza forestal en el territorio Twi Waupasa, Costa Caribe Norte de Nicaragua
}

\author{
Carlos Mendoza Blanco ${ }^{1}$ \\ Isay Talavera Lacayo ${ }^{2}$ \\ Keith Narváez Ismael ${ }^{3}$ \\ Enrique Cordón Suárez ${ }^{4}$ \\ Ronnie de Camino Velozo ${ }^{5}$
}

\section{Resumen}

El estudio se realizó con el fin de analizar la gobernanza forestal en el territorio Twi Waupasa, Municipio de Puerto Cabezas, Región Autónoma de la Costa Caribe Norte de Nicaragua. Se establecieron grupos focales y entrevistas individuales lo cual permitió identificar un conjunto de limitantes y problemas que interrumpen la gobernanza forestal; los conflictos internos entre comunidades vecinas, la mala distribución de los beneficios económicos, la invasión de tierra por colonos, el debilitamiento de las estructuras tradicionales por partidos políticos y la inexperiencia en el Manejo Forestal Comunitario, son situaciones que entorpecen la gobernanza. Para ello se ofrece un modelo de gobernanza forestal construido desde la perspectiva de los actores locales.

Palabras clave: Institucionalidad tradicional; participación; toma de decisiones.

\section{Abstract}

The study was conducted in order to analyze forest governance in Twi Waupasa territory, Puerto Cabezas Municipality, Autonomous Region of the North Caribbean Coast of Nicaragua. Focal groups and individual interviews were established which allowed identifying a set of constraints and problems that disrupt forest governance; Internal conflicts between neighboring communities, poor distribution of economic benefits, land invasions by settlers, weakening of traditional structures by political parties, and inexperience in Community Forest Management are situations that hinder governance. To this end, a forest governance model built from the perspective of local actors is offered.

Keywords: Traditional Institutional; participation; decision making.

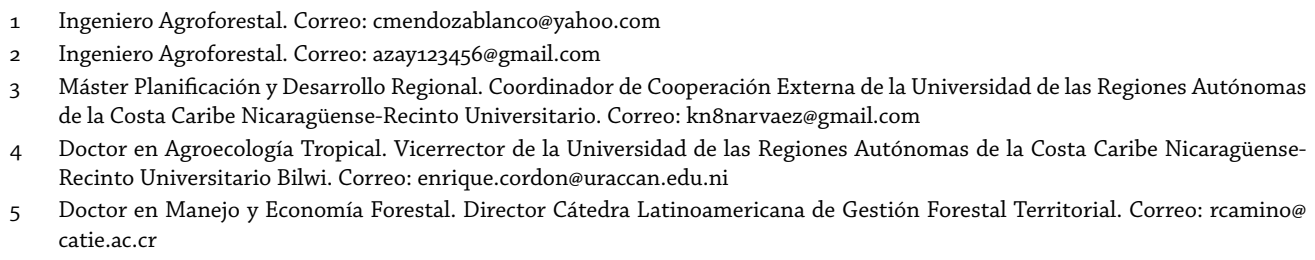

Recibido: 08/02/2017 Aprobado: 16/08/2017 


\section{Introducción}

En las últimas décadas se ha evidenciado que los gobiernos estatales, especialmente en los países en desarrollo no tienen la capacidad de ejercer una conservación responsable de los bosques, debido a diversas razones que generalmente les atañe la corrupción, en ese sentido se tiene mayor confianza en que los únicos que realmente pueden proteger los bosques son las mismas comunidades donde desarrollan sus estilos de vida para satisfacer sus necesidades fundamentales para el alivio a la pobreza.

Nicaragua posee una cobertura de vegetación forestal de 6.284,932 hectáreas, equivalentes al 53 por ciento del territorio terrestre nacional. Más del 70 en el porcentaje de los bosques existentes en el país se ubican en las regiones autónomas de la Costa Caribe (Thorslund, 2003), en territorios históricamente demandados y defendidos por los pueblos indígenas. El territorio indígena Miskitu Twi Waupasa, corresponde al municipio de Puerto Cabezas, Región Autónoma de la Costa Caribe Norte de Nicaragua, posee una extensión territorial de 125,249.46 ha, distribuidas en 15 comunidades.

Los comunitarios de este territorio están caracterizados por gozar de los patrimonios de sus recursos naturales, los cuales tienen la oportunidad de jugar papeles importantes como dueños de las tierras; sin embargo, posee pocas capacidades institucionales locales para impulsar la buena gobernanza forestal.

\section{Revisión de literatura}

\section{La gobernanza}

El termino gobernanza ocupa una connotación diversa para diferentes autores, pues para muchas personas este término suele parecer abstracto o confuso, aunque para otros puede parecer un término amplio con diversos componentes; pero, en realidad la conceptualización de la gobernanza puede ser adaptada a cada contexto de estudio.

Ante la diversa connotación conceptual, este estudio acoge el término Gobernanza adoptado por la Unión Internacional para la Conservación de la Naturaleza (UICN, 2013) considerado como el más adecuado. La gobernanza de los recursos naturales es un concepto que incluye normas, instituciones y procesos que determinan cómo se ejercen el poder y las responsabilidades, cómo se toman las decisiones y cómo participan los ciudadanos en el manejo de los recursos naturales. La gobernanza no es más que quién tiene la capacidad de ejercer el poder, influir, decidir; y cómo la toma de decisiones debe responder a obligaciones, responsabilidad, transparencia y rendición de cuentas. Es, por lo tanto, la relación articulada que mantienen actores, instituciones y normas o leyes. (Pazmiño, 2013). 


\section{RECURSOS NATURALES}

Para mejorar la gobernanza de los bosques es necesario un enfoque sistemático que identifique los puntos débiles, conciba e implemente soluciones oportunas, que dé seguimiento a los resultados, persiguiendo la continuidad, la adaptación y el aprendizaje para garantizar el progreso. Un marco analítico integral y ampliamente aceptado facilitará los esfuerzos, en función del mejoramiento de la gobernanza forestal (FAO, 2011).

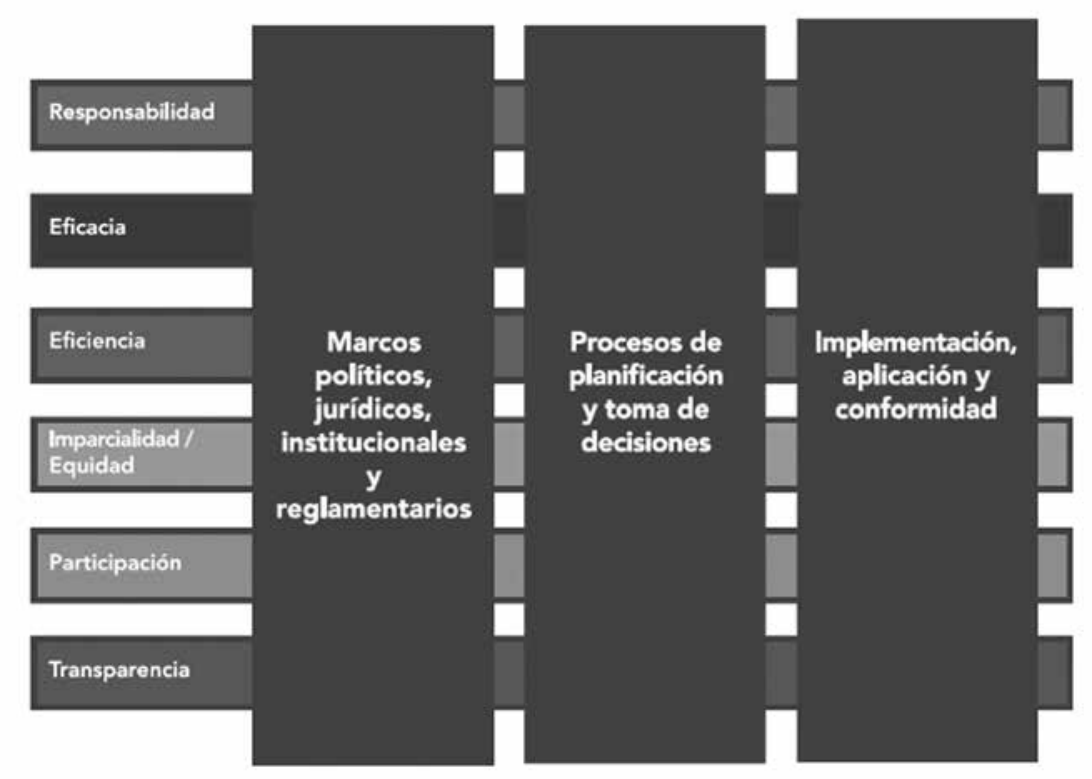

Figura 1: Pilares y principios de la gobernanza forestal (FA0, 2011).

Con el objetivo de la evaluación y seguimiento de la gobernanza, la FAO (2011), también propone una serie de componentes y subcomponentes para los tres pilares, además menciona que para el caso de los indicadores estos deben de ser construidos de acuerdo al contexto de cada país. Pese al nivel de investigación en el cual se ocupa el tema de gobernanza, este estudio no pretende ofrecer un análisis detallado y minucioso de todos los componentes, subcomponentes, construcción de indicadores, pilares y principios, pues para ello se requieren de mayores investigaciones de esta naturaleza. Pero, en efecto el estudio ofrece un análisis de manera implícita sobre el marco de evaluación y seguimiento de la gobernanza propuesto por la FAO.

\section{Estructura y funcionamiento de las comunidades indígenas}

La Asamblea Territorial. Es la mayor autoridad del territorio, está representada por todos los comunitarios, a la hora de reunirse únicamente se convoca a los representantes de cada una de las comunidades con el fin de establecer acuerdos y toma de decisiones para administrar la justicia y los recursos naturales del territorio. Estatuto del GTI Twi Waupasa (2011). En el caso de las Asambleas Comunales es diferente, en 
ella se reúnen todas las personas pertenecientes a esa comunidad, discuten y toman decisiones sobre a cuerdos para la gestión comunitaria las elecciones de los wihtas, síndico y los demás miembros, así mismo realizan asambleas a la hora de presentar un proyecto, o negociaciones con alguna empresa.

La Junta Directiva Territorial. Conocida también como gobierno territorial es la entidad ejecutiva y administrativa del territorio, su oficina se ubica en la cabecera municipal de Bilwi, en la que realizan el trabajo administrativo de cara a lograr la buena gobernanza. Está formada por el presidente, el vicepresidente, secretario, tesorero, un fiscal, un vocal, responsable de mujeres, un responsable de jóvenes, deporte y cultura, Responsable de Salud, Un responsable de Desarrollo, Un Sindico Territorial, Un Vocero o Divulgador del Territorio. La estructura y funcionamiento de los territorios indígenas, no se enmarca únicamente en las Juntas Directivas y sus funciones, sino que aglomera también un conjunto de normas locales para el uso acceso y disfrute de los recursos naturales, lo cual se relaciona con la institucionalidad tradicional y los mecanismos para la toma de decisiones a nivel territorial y comunal.

Las normas comunitarias son prácticas ancestrales apegadas a la cosmovisión indígena: todo cuanto existe, tiene vida, por lo tanto, existen normas de relación dadas a través de la ritualidad. De este modo, el territorio para nuestros pueblos es el hábitat donde se desarrolla la vida, se controla y manejan los recursos naturales sustentables a largo plazo (Pazmiño, 2007). En otro orden el funcionamiento de una institucionalidad tradicional resulta primordial dentro un territorio, pues permite tomar decisiones en consenso a través de los mecanismos tradiciones como las asambleas representativas. Una asamblea es "representativa" cuando hay opiniones predominantes sobre lo justo y lo injusto; sus miembros no deberían ser los portavoces de intereses particulares ni expresar la "voluntad" de una sección cualquiera particular Hayek (1973).

\section{Conflictos en la tenencia de la tierra en los territorios indígenas}

Los conflictos de tierra en los territorios de las comunidades indígenas se dan por la doble asignación de una misma parcela a diferentes personas, por desconocimiento en la titularidad de algún derecho de uso o usufructo sobre un determinado lote, por penetraciones ilegales, por robo de cosechas, por daño a la propiedad, por traslapes en los linderos y por transformación ilegal de la tierra comunal en propiedad privada (Bonilla, 2013). Estos conflictos impiden a que se desarrolle un manejo forestal. La Región del Caribe sirve como escenario de conflictos que ponen en riesgo el manejo forestal, hay pocos mecanismos idóneos de consulta que aseguren un pleno consentimiento libre por parte de las comunidades, de ahí se destacan los modelos de empresas que explotan el bosque.

Las actuales iniciativas para la promoción del Manejo Forestal Comunitario han dado inicio a procesos de ruptura en las relaciones de explotación de las comunidades 


\section{RECURSOS NATURALES}

por los madereros e intermediarios convencionales. No obstante, en su mayoría estas iniciativas tienen criterios técnicos y legales establecidos desde afuera, sin la efectiva participación y el control de las comunidades (Sabogal, 2008).

\section{Materiales y métodos}

El estudio fue de carácter cualitativo, orientado en conocer el estado de gobernanza forestal durante el 2015, se fundamentó en un proceso participativo con comunicación directa entre comunitarios de Twi Waupasa. Los métodos y técnicas de recopilación de información han sido la entrevistas con preguntas abiertas, grupo focales, revisión de estudios con relación al tema, revisión de referencias bibliográficas.

El universo comprendió la población del Territorio Twi Waupasa, que forma parte de un sistema de quince comunidades distribuida en 8 bloques. La muestra comprendió las comunidades de Maniwatla, Sukatpin, Yulo y Betel, por ser comunidades representativas del territorio. Se establecieron 3 grupos focales que fueron realizados en las comunidades de Maniwatla, Sukatpin y Betel, cada uno comprendió 10 integrantes con un total de 30 comunitarios consultados; así mismo se realizaron 4 entrevistas individuales con Autoridades Comunales que comprendió 2 entrevistas en Yulu y 2 en Maniwatla, consecuentemente se efectuaron 2 entrevistas individuales a Autoridades Territoriales y 4 entrevistas a Actores Claves a Nivel Regional: Como Miembros del CCF-A, Docentes, Instituciones. Con un total de 8 entrevistas individuales.

\section{Resultados y discusión}

\section{Estructura y funcionamiento del territorio Twi Waupasa}

Normas comunitarias para el uso y manejo de los recursos forestales. Existen diversas normas para el acceso a la utilización de los recursos en el ámbito de las comunidades, las cuales se han originado desde tiempos ancestrales, en donde dan cabida a una espiritualidad que poseen los comunitarios con los recursos. Pazmiño et al. (2007), sostiene que las normas contenidas en un territorio establecen el uso y manejo de los recursos que permite a cada elemento de la naturaleza existir.

Este manifiesto deja claro, de que las comunidades indígenas regulan el acceso a los recursos mediante normas tradicionales, las cuales descansan en la cosmovisión indígena que son las propias formas de interpretar e interactuar con la naturaleza. Un participante del grupo focal en Sukatpin, menciona lo siguiente:

Nosotros como ancianos pedimos permiso o perdón a los dueños de los árboles, por cortar una hoja, una rama o incluso todo el árbol, porque si no hacemos eso, los Dioses nos castigan con alguna enfermedad que a veces es imposible de curar si no buscamos los remedios a tiempo... 
Este manifiesto pone en razonamiento, que el uso de las normas tradicionales abona a la perpetuidad de los recursos, ya que incluyen procedimientos de sanciones ante el incumplimiento de las mismas, las cuales están apegadas a la cosmovisión indígena.

Estas normas hoy en día paulatinamente están siendo irrespetadas debido al cambio del modelo de relacionarse con el bosque que ha pasado de una visión de conservación a una visión de comercialización, lo cual esto se ha traducido en pérdida de los recursos forestales por satisfacer la demanda de recursos para empresas exógenas. Ante este escenario planteado, es urgente rescatar esos elementos que favorecen y consolidan la gobernanza forestal con una mirada de gestión colectiva fundamentada en los procesos de cumplimiento de normas tradicionales.

La Institucionalidad Tradicional: un camino hacia la gobernanza forestal. El Gobierno Comunal Indígena (GCI) y el Gobierno Territorial Indígena (GTI), son estructuras tradicionales del territorio, las cuales han sido ajustadas de acuerdo a las nuevas dinámicas y necesidades internas para mejorar su gobernanza. Ancestralmente los miembros de las instituciones tradicionales únicamente eran el wihta, el síndico, y el Consejo de Ancianos, actualmente el GTI está conformado por 12 miembros, lo cual esto deja como evidencia de que se han anexado en los últimos años nuevas figuras de cara a lograr la buena gobernanza forestal, $\mathrm{n}$ comunitario de Maniwatla en una entrevista explica lo siguiente:

Nuestros ancianos nos contaban que ellos antes no tenían tantas personas en la Junta Directiva, solamente estaban los ancianos, el wihta, y el índico, estos controlaban todo el territorio, pero ahora hay más integrantes y más problemas administrativos en nuestras Juntas Directivas.

De esta cita podemos comprender que la institucionalidad comunitaria no está funcionando bien, puesto que los problemas se están agudizando cada vez más. Por consecuencia es urgente enfrentar la acelerada distorsión del liderazgo. Proceso en el cual se evidencia una transculturización en los modos de liderazgo y ejercicio de la autoridad para la toma de decisiones. El tema de toma de decisiones es un impedimento para prosperar con la buena gobernanza forestal, puesto que desde hace décadas las comunidades viven bajo una informalidad en las asambleas representativas para tomar decisiones.

En este sentido, Hayek (1973), explica que en una asamblea representativa debe de haber opiniones predominantes sobre lo justo y lo injusto, sin depender de una decisión por grupos particulares. En este caso, la toma de decisiones en las asambleas se centraliza en un grupo de personas, en la cual el síndico, el wihta y el presidente son los que concentran todo el poder. Un participante del grupo focal realizado en Betel explica lo siguiente: 
A la hora de realizar alguna negociación los que andan más en movimiento son el síndico, el whihta y el presidente, nosotros casi no decimos nada porque nuestras decisiones son poco tomadas en cuenta...cuando llegamos a las asambleas ellos ya tienen todo planificado.

Esta cita evidencia que el tema de toma de decisiones se concentra en un grupo finito de actores que ejercen un poder parcial y autoritario, sobre todo cuando se trata de una decisión basada al manejo de los recursos económicos provenientes de alguna negociación relacionada al bosque, en la cual hay carencia de criterios de consenso, rendiciones de cuentas, transparencia y poca gestión colectiva.

\section{Principales limitantes y problemas de la gobernanza forestal en el territorio Twi Waupasa}

Conflictos externos. Alcanzar la seguridad en la tenencia de tierra se ha vuelto un dilema para los pueblos indígenas, dado que padecen de un fuerte fenómeno de invasión en sus territorios por personas ajenas que no poseen derechos colectivos, popularmente llamados colonos. Esta apropiación indebida de la tierra, crea una situación vulnerable que trastoca los fundamentos de convivencia directa que tienen los comunitarios con los recursos naturales. En ese particular una comunitaria de Sukatpin afirma lo siguiente.

... nosotros teníamos bastante tierra para la siembra, casería, leña entre otros... ahora las otras etnias (Colonos) vienen deteriorando nuestro bosque, ellos están destruyendo todo para la ganadería..."

La invasión de tierra, distorsiona los procesos de gobernanza forestal, puesto que los sistemas de producción enfocados en la ganadería con el rápido cambio de uso del suelo de bosque a pastizal dejan impactos negativos a futuro para la sobrevivencia, pues de esta manera se incrementa la deforestación, el cual atenta contra la economía comunitaria dado que se limita el acceso a la cacería, pesca, aprovechamiento de recursos maderables y no-maderables provocando la escases de recursos e interrumpiendo el desarrollo del Manejo Forestal Comunitario.

Esta problemática es el tema principal para las autoridades de este territorio, en donde consideran que la solución más factible es el saneamiento territorial. El saneamiento desde la percepción de los comunitarios es entendido como la manera de "limpiar" el territorio y que esté "puro", sin contaminación y sin destrucción alguna. Al respecto un miembro de la Junta Directiva Territorial menciona:

Nosotros tenemos que hacer una limpieza de nuestro territorio porque está muy invadida, y está siendo destruida [...] es por eso que les pedimos a los gobiernos que hagan ya, el saneamiento. 
Uno de los aspectos más anhelados por los territorios indígenas desde hace décadas, es la demarcación y titulación de sus tierras para efectuar la administración real de sus territorios. Para ello el Estado reconoce estos derechos históricos de los territorios indígenas, respondiendo a través de la Ley 445, Ley Régimen de Propiedad Comunal. En el Art. 45 de esta ley, sostiene que el proceso de demarcación y titulación cuenta con las etapas siguientes: 1). Etapa de presentación de solicitud; 2). Solución de conflictos; 3). Medición y amojonamiento; 4). La titulación; y 5). El saneamiento.

Cumplir esta última etapa ha sido el punto débil para el Estado, dado que se ve interpuesto a asumir errores estructurales en las políticas públicas que han padecido diferentes gobiernos de turno, además no le resultaría conveniente desalojar a un gran número de colonos-mestizos que en su mayoría son ex-combatientes quienes han defendido espontáneamente a lógicas partidarias durante la lucha armada ocurrida hace más de tres décadas. En ese particular Bonilla (2013), destaca que no se puede aislar o separar la etapa de saneamiento por razones de su complejidad o por intereses políticos, o partidarios.

Es notoria la incapacidad e insuficiente voluntad del Estado en materializar la etapa de saneamiento, puesto que para realizar esta labor se requiere de mucha precisión y economía. A pesar de que existen varias metodologías para solventar el conflicto; como la negociación, indemnización, mediación, judicialización, la convivencia y desalojos, los comunitarios exigen más a que sea el desalojo la solución, dado que tienen un profundo resentimiento por los daños irreversibles causados al bosque, tanto así que ha trastocado sus estilos de vida atentando contra sus medios de subsistencia. En otro orden, seria parcial e inconcluso dejar a los colonos como los únicos actores que causan conflictos externos en el territorio, cuando además existe la directa injerencia de los partidos políticos que socavan y debilitan a las estructuras locales. Para ello han montado estructuras dentro del territorio, que contribuyen al desarrollo de las comunidades, pero a veces existe un sinnúmero de intereses políticos y económicos.

Algunos miembros de estas estructuras políticas deslegitiman las estructuras locales comunitarias, anteponiendo los intereses políticos ante los beneficios comunitarios. En consecuencia, con lo anterior, un comunitario de Maniwatla menciona lo siguiente:

Nosotros estamos muy preocupados...este gobierno, ha puesto CLS en la comunidad... ahora estos son los quieren manejar a los ancianos, a los Wihtas, incluso a los policías comunales.

En este particular los Consejos de Liderazgo Sandinista (CLS), son estructuras que trabajan en función de resolver problemas de seguridad, transporte, educación, deporte, cultura y extienden los programas de gobierno como el plan techo, los bonos productivos, entre otros. Estas estructuras apoyan en cierta medida al territorio, pero 


\section{RECURSOS NATURALES}

es un hecho lo que sostiene el comunitario, que algunos de estos miembros quieren suponer un poder autoritario, esta condición deslegitima la acción solidaria que hace el gobierno de turno y además se corre el riesgo a una transculturización.

Experiencias de Manejo Forestal Comunitario en el Territorio Twi Waupasa. Aprovechar el bosque como un capital natural oportuno para fomentar un bienestar social es el principal reto para las comunidades indígenas, es ahí donde el Manejo Forestal Comunitario resulta importante para que los comunitarios tengan un dominio real de sus bosques promoviendo un desarrollo económico, pero sin dejar atrás la conservación de la naturaleza. Sabogal (2008), destaca que el manejo forestal comunitario es la opción más promisoria para resolver el gran dilema de la conciliación entre la preservación de la naturaleza y el desarrollo económico.

Para ello el Manejo Forestal Comunitario no debe de verse únicamente como la simple forma de comercializar los productos maderables en mercados locales a como tradicionalmente se ha practicado, sino que debe de ser una oportunidad para establecer pequeñas y medianas Empresas Forestales Comunitarias (EFC), con una obra gerenciada por los propios protagonistas locales con la capacidad de ejercer el poder ante la toma de decisiones, sin permitir una socavación externa que debilite la gobernanza forestal. En ese particular desde hace décadas se han dado los primeros pasos de cara a la conformación de las EFC en la Costa Caribe Norte de Nicaragua; no obstante, tras el paso del huracán Félix, se aceleró esta oportunidad a través de la donación de aserríos portátiles que hizo el gobierno regional para el aprovechamiento de la madera caída para la construcción de viviendas como primera etapa de uso, y la creación de EFC como etapa ulterior.

Después del paso del huracán Félix hubo un masivo proceso de fortalecimiento de los gobiernos comunales que comprendió entre 2006-2008, el territorio Twi Waupasa logró apropiarse de un aserrío portátil LT 40, el cual fue donado por el Gobierno Nacional en coordinación con el Gobierno Regional, exclusivamente para que se lleve a cabo el aprovechamiento de los árboles afectados por el ciclón.

El aserradero que se extendió al territorio de carácter comunitario, tenía como objetivo beneficiar de forma directa a las 15 comunidades del territorio, aprovechando los árboles caídos para ser procesados y usarlos como construcción de viviendas para los comunitarios con mayor necesidad; sin embargo, la incipiente administración del aserradero provocó la pérdida de la misma ya que se había acordado usarse de manera rotativa para todas las comunidades, pero fue todo lo contrario. Un comunitario de la comunidad de Maniwatla explica lo siguiente:

A partir del 2008, el Gobierno Regional nos donó un aserrío para la construcción de viviendas, este tenía que usarse de manera rotativa..., primero Maniwatla luego Yulu, Truhlaya, y así sucesivamente, pero no se hizo nada ya que el Síndico solo 
comercializaba Madera... y por último en el 2011 lo llevó a Rosita y lo vendió por 20,000 dólares.

La pretensión de algunos líderes que llegan al poder del Gobierno Comunal ha causado ciertas confrontaciones que después de su período deja mucho por cuestionar, no han cumplido con todas las expectativas que los comunitarios esperaban, no realizaban ninguna evaluación respecto al uso y manejo del aserrío.

El aserrío fue usado de manera personal, todos los ingresos monetarios fueron mal administrados por tal razón no se siguió haciendo uso de sus servicios, y en consecuencia no se construyó ninguna vivienda lo cual las comunidades siempre siguen con las mismas necesidades. En otro orden, otra experiencia que ha tenido el territorio específicamente la comunidad de Maniwatla sobre Manejo Forestal Comunitario fue con la negociación con una empresa Estatal denominada Alba Forestal. En este sentido la creación de Alba-Forestal por parte del estado, fue con la búsqueda de un mejor aprovechamiento de la madera tumbada por el huracán Félix, en donde supuestamente las comunidades tendrían ingresos económicos justos y una mayor integración en la cadena productiva forestal, para ello esta empresa ha venido negociando con diferentes comunidades de la Costa Caribe Norte de Nicaragua.

Pese a la poca capacidad económica, técnica y empresarial que tienen las comunidades junto con el tedioso proceso burocrático al cual tienen que atenerse, les resulta difícil prosperar con la conformación de sus propias EFC. Aunque esta situación resulte una limitante para las comunidades indígenas, en contraste con las empresas forestales externas es una oportunidad para penetrar con mayor facilidad al territorio puesto que poseen mayor poder económico y mayores capacidades empresariales y administrativas como es el caso de Alba-Forestal.

Al consultar con el ex-juez de Maniwatla sobre la negociación que sostuvieron con Alba-Forestal, explicó lo siguiente:

Alba-Forestal vino y nos ofreció 15 dólares por cada $\mathrm{M}^{3}$ extraído, lo cual extrajeron un total de $1,890 \mathrm{M}^{3}$, nosotros aceptamos ya que ellos pusieron sus maquinarias, hicieron su Plan de Manejo, pagaron a INAFOR, o sea ellos hicieron todo, nosotros no hicimos nada más que agarrar el dinero.

Este tipo de negociación se da muy a menudo en las comunidades indígenas, puesto que de esta manera se ahorran lidiar con el difícil proceso burocrático; sin embargo, aunque parezca exitosa esta negociación en un primer momento, también ofrece un conjunto de limitantes: 


\section{RECURSOS NATURALES}

- La negociación efectuada disminuye la tendencia a que los comunitarios se independicen y formen su propia EFC, pues hay una posición muy acomodada conformista y dependiente.

- Existe la limitada participación de todos los comunitarios en la toma de decisiones, pues los mismos dirigentes que mal administraron el aserrío portátil fueron los mismos quienes entablaron la negociación con Alba-Forestal, restringiendo de esta manera derechos de consenso colectivo.

- El limitado rol protagónico de los comunitarios para llevar a cabo el Manejo Forestal permite mayor socavación de empresas externas provocando poca inserción de las comunidades en la cadena productiva forestal.

La nueva dinámica de negociación con términos empresariales trastoca inevitablemente los modelos institucionales endógenos, puesto que se enfrentan a situaciones que no conocen como el caso respecto del tema de la negociación con empresas exógenas, requieren por ende hacer ajustes institucionales con mayores capacidades administrativas y de liderazgo; pero, sin perder el enfoque sistémico de bosque que descansa en la cosmovisión comunitaria. Así mismo requieren de un permanente proceso de acompañamiento del gobierno que contribuyan a sintetizar el tedioso proceso burocrático, ya que según el sentir de los comunitarios es muy prolongado, costoso y termina por otorgar los derechos de usufructo a empresas exógenas.

\section{Modelo de Gobernanza Forestal para el Territorio Twi Waupasa}

Con el fin de responder a la sostenibilidad y a la buena gobernanza forestal, hacemos la siguiente propuesta que responde a una alternativa mediante una gráfica que consiste en una ruta crítica, en la cual demuestra cómo debe de llevarse a cabo el proceso de gobernanza forestal a nivel del territorio. 


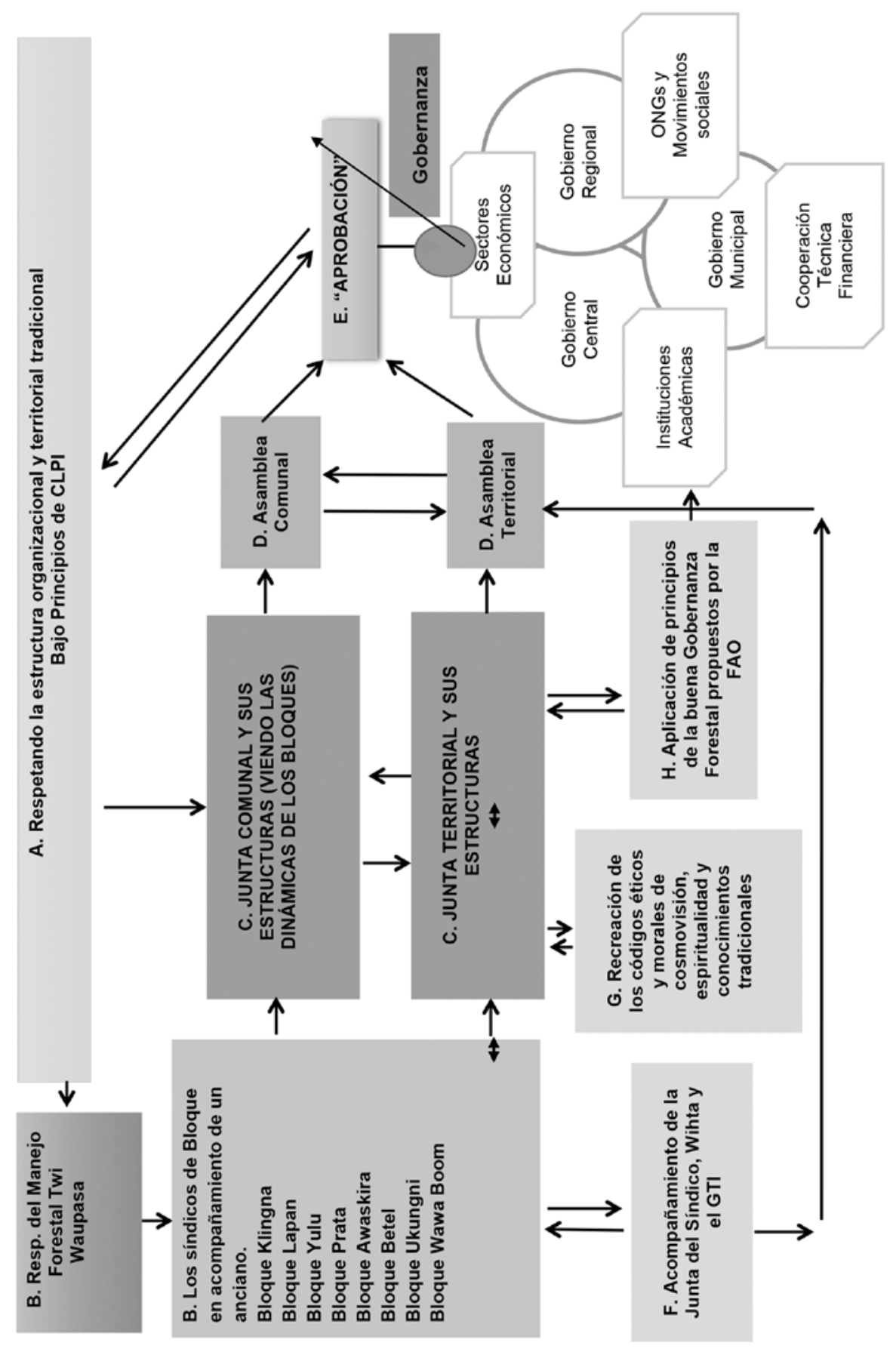

Figura 1: Modelo de gobernanza forestal a nivel del territorio de Twi Waupasa 2015 


\section{Relación de los componentes de la Ruta Crítica para la buena Gobernanza Forestal de Twi Waupasa}

Desde la perspectiva de los actores locales, los procesos de gobernanza forestal en el territorio, tienen una serie componentes articuladores, su dinamismo brinda un escenario equilibrado de oportunidades para mejorar las condiciones de vida de las comunidades que son dueñas de los bosques.

En primer lugar se inicia con el respeto al principio del Consentimiento Libre Previo e Informado (CLPI), cualquier intervención por actores externos deben de respetar esos derechos; así mismo los comunitarios deben de procurar a que se cumpla, para luego poder entablar una negociación con los responsables del manejo forestal; que en este caso son los síndicos de cada bloque a través del acompañamiento de los ancianos, en consecuencia la Junta Directiva Comunal y Territorial: deben de respetar el CLPI y las dinámicas de los bloques para llevar a cabo el manejo forestal.

Posteriormente a través de una Asamblea Comunal o Territorial; debe de darse la aprobación con el pleno acompañamiento de la estructura del síndico, el wihta, y el GTI, tomando en cuenta los códigos éticos y morales que descansa en la cosmovisión comunitaria y apropiándose de los Principios de la buena Gobernanza Forestal. La aprobación también se vincula con el acompañamiento de los diferentes niveles de gobierno y sectores de la gobernanza forestal, y por último la articulación e interrelación de todos los componentes es la convergencia que da lugar a la gobernanza forestal del territorio Twi Waupasa.

\section{Conclusiones}

El territorio Twi Waupasa posee sus estructuras organizativas de gobierno, entre ellas: las Juntas Directivas Comunales - Territoriales y las Asambleas Comunales Territoriales, estas presentan problemas de funcionamiento en el cumplimiento de las funciones de acuerdo al estatuto de gobierno y además hay poca implementación de los principios de la buena gobernanza forestal.

El conjunto de normas locales contenidas en el territorio garantiza la gestión efectiva de los recursos naturales; sin embargo, las nuevas generaciones están irrespetando estas normas. Los conflictos externos en el territorio, descansan en la apropiación de tierra por personas ajenas a los territorios que no comparten derechos colectivos, en este sentido la presencia de colonos deja impactos negativos a futuro.

La instauración de nuevas estructuras dentro del territorio pone en riesgo el desarrollo de una transculturización. El proceso burocrático para llevar a cabo el manejo forestal comunitario es muy prolongado, costoso y complejo, lo que termina por otorgar derechos de usufructo a empresas exógenas, de ahí que sea necesario un 
acompañamiento pleno por parte del gobierno regional que ayuden a sintetizar el tedioso proceso burocrático.

El modelo de gobernanza forestal propuesto, constituye una ruta crítica que se debe seguir, lo cual inicia con el respeto al principio del CLPI; posteriormente, los responsables del Manejo Forestal deben de ser los síndicos en acompañamiento de consejeros; luego, mediante una asamblea comunal o territorial se deben de dar a conocer las decisiones y acuerdos para que posteriormente se dé la aprobación con el acompañamiento de los GTI y los demás actores, respetando la cosmovisión comunitaria y aplicando los principios de la buena gobernanza forestal.

\section{Lista de referencias}

Asamblea Nacional de Nicaragua. (2003). Ley (445) del régimen de propiedad comunal de los pueblos indígenas y comunidades étnicas de las regiones autónomas de la costa atlántica de Nicaragua y de los ríos Bocay, Coco, Indio y Maíz. Managua, Nicaragua.

Bonilla, W. (2013). Diagnóstico sobre el Saneamiento de los Territorios Indígenas y étnicos de la RAAN, Nicaragua. Cooperativa de Profesionales MASANGNI R.L.

Estatuto de Gobierno (2011). Territorio Indígena de Twi Waupasa, Región Autónoma de la Costa Caribe Norte, Bilwi Puerto Cabezas. RACCN.

FAO (2011). Marco para la evaluación y seguimiento de la gobernanza forestal. Roma, Italia (Programa sobre los bosques (PROFOR).

Hayek, F. (1973). Libertad económica y gobierno representativo. The Institute of Economic Affairs, Londres.

Narváez, K. (2014). Plan de Ordenamiento Forestal Territorio Twi Waupasa, Bilwi, Costa Caribe de Nicaragua: URACCAN

Pazmiño, A. (2007). Mujeres Indígenas, territorialidad y biodiversidad en el contexto latinoamericano. Colombia.

Pazmiño A. (2013.) Aprendiendo sobre la gobernanza. Conservación Ahora. Revista electrónica de la UICN.

Sabogal, C. (2008). Manejo forestal comunitario en América Latina. Experiencias, lecciones aprendidas y retos para el futuro. Sabogal, C.; W., J.d.; Pokorny, B.; Louman, B. eds. Bogor, Indonesia, Centro para la Investigación Forestal (CIFOR).

Thorslund, O. (2003). Estudio Sectorial Forestal de las Regiones Autónomas del Atlántico Norte y Sur de Nicaragua. Corredor Biológico del Atlántico, MARENA. 